Pub. 548

\title{
Redução da concentração alveolar mínima (CAM) em cães anestesiados com isoflurano associado a fentanila
}

\author{
Minimun alveolar concentration (MAC) reduction in dogs anesthetized with isoflurane \\ combined with fentanyl
}

\author{
Isabella Dib Ferreira Gremião ${ }^{1}$, Amary Nascimento J r. ${ }^{2}$, J oão Henrique Neves Soares ${ }^{1}$, \\ Fábio Otero Ascoli ${ }^{1} \&$ Firmino Mársico Filho ${ }^{3}$
}

\begin{abstract}
RESUMO
A utilização transoperatória de opióides previne as respostas autonômicas ao estímulo doloroso resultando em melhor estabilidade hemodinâmica, reduz as exigências de agentes anestésicos intravenosos ou inalatórios como também a dor e o desconforto no pós-operatório. Este estudo teve como objetivo avaliar o efeito de uma dose de fentanila em infusão contínua na CAM do isoflurano em cães. Para tanto, foram utilizados seis cães, fêmeas, sem raça definida, com peso variando entre dez e quinze $\mathrm{kg}$, na faixa etária entre dois e quatro anos. No primeiro episódio anestésico (G1), a CAM do isoflurano foi determinada de forma duplicada em cada animal. Após determinação da CAM, foi administrada fentanila na dose de $30 \mu \mathrm{g} / \mathrm{kg}$ durante 20 minutos seguido por infusão contínua na velocidade de $0.2 \mu \mathrm{g} / \mathrm{kg} / \mathrm{min}$. Decorridos 60 minutos de infusão, a CAM isoflurano-fentanila foi determinada de forma duplicada em cada animal. No segundo episódio anestésico (G2), cada animal recebeu solução salina administrada numa dose inicial durante 20 minutos seguida por infusão contínua, utilizando-se o mesmo volume do fentanila. A CAM do isoflurano foi igualmente determinada de forma duplicada. Observou-se que a fentanila reduziu significativamente ( $\mathrm{P}<0,05)$ a CAM do isoflurano de $1.27 \pm 0.02 \%$ para $0.73 \pm 0.08 \%$, representando uma redução de $42 \%$. A CAM isoflurano-solução salina não foi significativamente diferente da CAM do isoflurano $(\mathrm{P}>0,05)$. Através dos resultados obtidos, foi possível concluir que a administração de fentanila na dose de $0.2 \mu \mathrm{g} / \mathrm{kg} / \mathrm{min}$ em infusão contínua reduz significativamente $(\mathrm{P}<0,05)$ a CAM do isoflurano, resultando em uma redução na necessidade do agente inalatório no período transanestésico.
\end{abstract}

Descritores: opióides, fentanila, isoflurano, cães, CAM.

\section{ABSTRACT}

The surgical use of opioids eliminates autonomic responses to pain stimulus, which stabilizes the hemodynamics, reduces the required dose of intravenous or inhalatory anesthetics during surgery, and minimizes the postsurgical discomfort experienced by patients. The purpose of this study was to evaluate the effect of a constant-rate infusion of fentanyl on minimum alveolar concentration (MAC) of isoflurane in dogs. Six healthy female mongrel dogs, with an average weight between ten and fifteen $\mathrm{kg}$ and age between two and four years, were used. In the first anesthetic episode (G1), isoflurane MAC was determined in duplicate in each animal. Following MAC determination, a loading dose $(30 \mu \mathrm{g} / \mathrm{kg})$, administered over a period of 20 minutes, and a maintenance infusion $(0.2 \mu \mathrm{g} / \mathrm{kg} / \mathrm{min})$ of fentanyl citrate were both initiated at the same time. Isoflurane-fentanyl MAC was determined in duplicate in each animal 60 minutes after the onset of infusion. In the second anesthetic episode (G2), each animal received saline solution administered as a loading dose during 20 minutes followed by a constant-rate infusion, using volumes equal to those used for fentanyl. Isoflurane MAC was determined (in duplicate) 60 minutes after the onset of infusion. Fentanyl significantly decreased isoflurane $\mathrm{MAC}(\mathrm{P}<0.05)$ from $1.27 \pm 0.02 \%$ to $0.73 \pm 0.08 \%$, representing a reduction of $42 \%$. Isofluranesaline solution MAC did not differ significantly from isoflurane-fentanyl MAC $(\mathrm{P}>0.05)$. This result allows us to conclude that the administration of a constant-rate infusion of fentanyl $(0.2 \mu \mathrm{g} / \mathrm{kg} / \mathrm{min})$ significantly decreased isoflurane MAC, allowing a reduction in the requirements of the inhalant agent during the perioperative period.

Key words: opioids, fentanyl, isoflurane, dogs, MAC.

${ }^{1}$ Curso de Pós-Graduação em Medicina Veterinária da Faculdade de Veterinária (FAVET) da Universidade Federal Fluminense (UFF). ${ }^{2}$ Disciplina de Técnica Cirúrgica e Anestesiologia Veterinária FAVET/UFF. ${ }^{3}$ Pesquisador do CNPq.

CORRESPONDÊNCIA: A. Nascimento Jr. [e-mail: amary@urbi.com.br] Rua Vital Brazil Filho 64; 24230-340 Niterói, RJ - Brasil. 


\section{INTRODUÇÃO}

Os agentes anestésicos intravenosos são muito utilizados durante anestesia inalatória, pois algumas propriedades como hipnose, analgesia, relaxamento muscular e abolição dos reflexos autonômicos são alcançados com menor concentração do anestésico inalatório, evitando-se aprofundamento do plano anestesiológico e, conseqüentemente, depressão cardiovascular e respiratória. Esta técnica é conhecida como anestesia balanceada [9].

O isoflurano (Forane ${ }^{\circledR}$ ) foi introduzido para uso clínico em 1981 e desde então se tornou um dos anestésicos inalatórios mais amplamente utilizados por apresentar vantagens significativas sobre seus precedentes [13]. A potência anestésica do isoflurano é intermediária a do halotano e a do enflurano [12].

A concentração alveolar mínima (CAM) foi conceituada como a concentração de um anestésico inalatório no alvéolo, a uma atmosfera, capaz de inibir movimentos intencionais em 50\% dos pacientes expostos a um estímulo doloroso supramáximo e, portanto, corresponde à dose efetiva ${ }_{50}\left(\mathrm{D}_{\mathrm{E}}{ }_{50}\right)$, em que metade dos indivíduos estará anestesiada e a outra metade ainda não terá alcançado tal estado [21]. Segundo estes autores, a CAM é inversamente proporcional à potência, isto é, quanto menor a CAM, maior será a potência de um anestésico volátil.

$\mathrm{O}$ valor da CAM do isoflurano não varia em função da duração da anestesia. Entretanto, diminui quando o anestésico é associado ao óxido nitroso, opióides, agonistas adrenérgicos $\alpha_{2}$, benzodiazepínicos, alguns representantes do grupo dos fenotiazínicos, barbitúricos e agentes dissociativos [12, 21].

Os opióides são os fármacos mais utilizados como parte de uma técnica anestésica balanceada. A utilização transoperatória de opióides previne as respostas autonômicas ao estímulo doloroso resultando em melhor estabilidade hemodinâmica, reduz as exigências de agentes anestésicos intravenosos ou inalatórios como também a dor e o desconforto no pósoperatório [9].

A fentanila $\left(\right.$ Fentanil $\left.^{\circledR}\right)$, sob a forma de citrato, é um opióide sintético 100 a 300 vezes mais potente que a morfina [24] e 500 vezes mais potente que a meperidina [11]. É indicada como medicação préanestésica, anestésico primário, em combinação com o droperidol para neuroleptoanalgesia ou como adju- vante na anestesia inalatória para controle da dor transoperatória e controle de respostas autonômicas ao estímulo doloroso [5,20].

As doses de fentanila em cães variam de 0.04 a $0,08 \mathrm{mg} / \mathrm{kg}$ IM, SC ou IV [22]. A administração de fentanila em bolus IV (1 a $5 \mu \mathrm{g} / \mathrm{kg}$ ) seguido por infusão contínua (1 a $5 \mu \mathrm{g} / \mathrm{kg} / \mathrm{hora}$ ) fornece boa sedação e analgesia no pós-operatório [5]. Clinicamente, infusão contínua de fentanila é menos efetiva em animais que não receberam o bolus de fentanila. Ilkiw [9] recomenda um bolus de $10 \mu \mathrm{g} / \mathrm{kg}$ seguido de uma infusão contínua de $0.7 \mu \mathrm{g} / \mathrm{kg} / \mathrm{min}$ para se obter uma concentração plasmática de fentanila de aproximadamente $28 \mathrm{ng} / \mathrm{mL}$, pois já foi demonstrado que uma concentração plasmática maior que $30 \mathrm{ng} / \mathrm{mL}$ não produziu mais que $65 \%$ de redução na CAM do enflurano em cães. Hellyer et al. [6] recomendam a utilização de fentanila administrada em bolus $(10 \mu \mathrm{g} / \mathrm{kg}$ durante 60 segundos) seguida por infusão contínua $(0.3 \mu \mathrm{g} / \mathrm{kg} / \mathrm{min})$ em cães.

Nas numerosas espécies animais, estudos documentam que os opióides reduzem a CAM do anestésico inalatório necessário para prevenir resposta a um estímulo doloroso [3,4,6,8,10,14,16-18,26-28]. Sabendo-se que os opióides produzem uma redução dose-dependente da CAM dos anestésicos inalatórios [16], o objetivo deste estudo foi avaliar o efeito de uma dose de fentanila, administrada através de infusão contínua, na CAM do isoflurano em cães.

\section{MATERIAIS E MÉTODOS}

Animais - Foram utilizados 6 cães, fêmeas, hígidas, sem raça definida (srd), com peso variando entre $10 \mathrm{e}$ $15 \mathrm{~kg}$, na faixa etária entre 2 e 4 anos, oriundos do canil da Faculdade de Veterinária da Universidade Federal Fluminense. Os animais foram previamente vermifugados, vacinados e submetidos a ovariohisterectomia. Todos os animais foram submetidos a exames clínico e laboratorial - hemograma completo e bioquímica (uréia, creatinina, TGO, TGP, glicose e proteínas totais). Constatada a higidez dos animais antes da realização do experimento, os mesmos foram submetidos a jejum alimentar de 12 horas e água ad libitum.

Instrumentação - Este estudo foi conduzido de acordo com as normas de vivissecção de animais descritas pelo Colégio Brasileiro de Experimentação Animal (COBEA) sob a lei $\mathrm{n}^{\circ}$ 6.638, de 8 de maio de 1979. 
Todos os procedimentos experimentais foram realizados no mesmo horário a fim de evitar possíveis alterações na mensuração da CAM devido às alterações circadianas. Inicialmente, os animais foram anestesiados com isoflurano ${ }^{1}$ a $4 \%$ sob um fluxo de oxigênio de $5 \mathrm{~L} / \mathrm{min}$, administrado através de uma máscara facial ${ }^{2}$ conectada a um circuito valvular com absorção de $\mathrm{CO}_{2}{ }^{3}$ utilizando-se um vaporizador calibrado ${ }^{4}$ para isoflurano. Após a indução, realizou-se a intubação orotraqueal e os animais foram mantidos em ventilação espontânea. Durante a instrumentação, o fluxo de oxigênio foi reduzido para $2 \mathrm{~L} / \mathrm{min}$ e a concentração de isoflurano expirada mantida entre 2,5 e $3 \%$. Os animais foram posicionados em decúbito lateral direito. Utilizando-se uma técnica asséptica, a veia cefálica foi cateterizada com dispositivo intravenoso $20 \mathrm{G}$ para a administração de solução fisiológica $\mathrm{NaCl}$ a $0,9 \%$, no volume de $15 \mathrm{~mL} / \mathrm{kg} /$ hora. Em seguida, realizou-se assepticamente a cateterização da artéria metatarsiana dorsal com dispositivo intravenoso $22 \mathrm{G}$. Este acesso permitiu a coleta de amostras de sangue arterial assim como a mensuração contínua das pressões arteriais sistólica (PAS), média (PAM) e diastólica (PAD). Esta mensuração foi realizada através de um transdutor de pressão ${ }^{5}$ previamente zerado e calibrado antes de cada estudo. Os gases sanguíneos arteriais foram mensurados durante as aferições da CAM, antes de cada estímulo, para assegurar que seus valores estivessem dentro dos limites normais de $\mathrm{pH}(7,35-7,45)$, pressão arterial parcial de oxigênio $\left(\mathrm{PO}_{2}\right)(>90 \mathrm{mmHg})$ e pressão arterial parcial de dióxido de carbono $\left(\mathrm{PCO}_{2}\right)(35-$ $45 \mathrm{mmHg}$ ). O sangue arterial foi coletado de forma anaeróbia por meio de seringas de vidro previamente heparinizadas, armazenado em gelo e processado num tempo máximo de 30 minutos através de um hemogasômetro. ${ }^{6}$ Com a finalidade de evitar a perda de calor, foi utilizado um colchão térmico, ${ }^{7}$ sendo a temperatura $\left(37,5-38,5^{\circ} \mathrm{C}\right)$ mensurada através de um termômetro digital, ${ }^{8} \mathrm{com}$ seu sensor posicionado na porção torácica do esôfago. A frequiência cardíaca e o ritmo cardíaco foram avaliados durante todo o experimento através de um eletrocardioscópio ${ }^{9}$ na derivação II do plano frontal. A saturação de $\mathrm{O}_{2}\left(\mathrm{SpO}_{2}\right)$ foi determinada pelo uso de um oxímetro de pulso. ${ }^{10}$ Amostras de gases expirados foram utilizadas para mensuração da concentração de gás anestésico e determinação da concentração alveolar mínima (CAM) do anestésico. As amostras foram coletadas com seringas de vidro de $10 \mathrm{~mL}$, durante 3 expirações consecutivas, através de um cateter de nylon posicionado na porção distal do tubo endotraqueal. A concentração de isoflurano expirada assim como a pressão parcial de $\mathrm{CO}_{2}$ ao final da expiração $\left(\mathrm{ETCO}_{2} \mathrm{mmHg}\right)$ foram mensurados através de um analisador de gases exalados, ${ }^{11}$ o qual foi calibrado com gás próprio para isoflurano antes e durante a realização de cada experimento.

Procedimento - Para maior homogeneidade deste trabalho, cada animal foi submetido a 2 tratamentos distintos, com um intervalo de uma semana entre cada estudo. Os tratamentos consistiram na administração de fentanila e solução salina. Apenas um tratamento foi administrado durante cada estudo. No primeiro episódio anestésico (G1), a CAM do isoflurano foi determinada de forma duplicada. Após determinação da CAM, foi administrada fentanila ${ }^{12}$ na dose de $30 \mu \mathrm{g} / \mathrm{kg}$ durante 20 minutos seguida por infusão contínua na velocidade de $0,2 \mu \mathrm{g} / \mathrm{kg} / \mathrm{min}$ [16] através de uma bomba de infusão contínua. ${ }^{13}$ Decorridos 60 minutos de infusão, a CAM isoflurano-fentanila foi determinada de forma duplicada em cada animal. No segundo episódio anestésico (G2-Grupo Controle), com a CAM do isoflurano previamente determinada, foi administrada solução salina durante 20 minutos (mesmo volume de fentanila) e em infusão continua (mesmo volume e velocidade de fentanila). Decorridos 60 minutos, a CAM isoflurano-solução salina foi igualmente determinada de forma duplicada.

Determinação da CAM - A CAM do isoflurano foi determinada utilizando-se a técnica descrita por Eger $e t$ $a l$. [2]. Após instrumentação e com os animais respirando espontaneamente, a concentração expirada de isoflurano foi ajustada com base na profundidade da anestesia e mantida constante durante 15 minutos com o objetivo de permitir um equilíbrio entre as pressões parciais de anestésico nos alvéolos, sangue arterial e cérebro.

Um estímulo de corrente elétrica (50 volts/50 ciclos/seg) durante 60 segundos foi aplicado por meio de um estimulador elétrico ${ }^{13}$ através de agulhas hipodérmicas de aço posicionadas subcutaneamente na face posterior da região do tarso. Foi considerado como resposta positiva um movimento muscular grosseiro, geralmente da cabeça ou das extremidades. Tosse, deglutição, mastigação ou alterações simpáticas não foram consideradas respostas positivas. O estímulo elétrico foi descontinuado diante de uma resposta positiva 
detectada antes que se completasse 1 minuto de estimulação. A concentração anestésica foi aumentada (após uma reposta positiva) ou diminuída (após uma resposta negativa) em 10 a $20 \%$ de acordo com a resposta ao estímulo, sendo que, antes de cada estímulo, a concentração expirada de isoflurano foi mantida constante por 15 minutos com o objetivo de permitir um equilíbrio entre as pressões parciais de anestésico nos alvéolos, sangue arterial e cérebro. Após determinação da CAM do isoflurano, foi iniciada a infusão contínua com fentanila, sendo os animais ventilados mecanicamente para que os valores de $\mathrm{PaO}_{2}$ e $\mathrm{PaCO}_{2}$ fossem mantidos dentro dos limites de normalidade. Ao mesmo tempo em que se iniciou a infusão contínua, a concentração de isoflurano foi reduzida e mantida constante por 60 minutos, sendo a CAM isoflurano-fentanila igualmente determinada de forma duplicada.

A CAM foi determinada individualmente, de forma duplicada, como a média da mais alta concentração que permite um movimento grosseiro depois de submetido ao estímulo e a mais baixa concentração que previne uma resposta positiva. Variáveis monitoradas (FC, FR, PAS, PAM e PAD, pH, $\mathrm{PaO}_{2}, \mathrm{PaCO}_{2}$, concentração expirada de isoflurano, $\mathrm{ETCO}_{2}, \mathrm{CO}_{2}$ reabsorvido, $\mathrm{SpO}_{2}$ e temperatura corporal) foram obtidas antes de cada estímulo. Os resultados obtidos foram analisados estatisticamente pelo teste de Wilcoxon para amostras pareadas. Significância foi definida como valores de $\mathrm{P}<0,05$.

\section{RESULTADOS}

Tabela 1. Concentração alveolar mínima (\%) do isoflurano em seis animais. Letras diferentes na mesma linha diferem estatisticamente $(P<0,05)$ pelo teste de Wilcoxon

\begin{tabular}{lccc}
\hline & Isoflurano & $\begin{array}{c}\text { Isoflurano - } \\
\text { Fentanil }\end{array}$ & $\begin{array}{c}\text { Isoflurano - } \\
\text { Salina }\end{array}$ \\
\hline Animal 1 & 1,28 & 0,76 & 1,29 \\
Animal 2 & 1,26 & 0,66 & 1,24 \\
Animal 3 & 1,24 & 0,63 & 1,25 \\
Animal 4 & 1,29 & 0,85 & 1,27 \\
Animal 5 & 1,25 & 0,76 & 1,24 \\
Animal 6 & 1,29 & 0,73 & 1,29 \\
X \pm DP & $1,27 \pm 0,02^{\mathrm{A}}$ & $0,73 \pm 0,08^{\mathrm{B}}$ & $1,26 \pm 0,02^{\mathrm{A}}$ \\
\hline
\end{tabular}

Tabela 2. Parâmetros cardiopulmonares e hemogasométricos obtidos à partir de seis animais (média \pm desvio padrão) durante a determinação da CAM. Letras diferentes na mesma coluna diferem estatisticamente pelo teste de Wilcoxon $(P<0,05)$.

\begin{tabular}{|c|c|c|c|c|c|c|c|c|c|c|c|c|}
\hline & $\begin{array}{l}\text { F.C. } \\
\text { b.p.m. }\end{array}$ & $\begin{array}{c}\text { F.R. } \\
\text { m.p.m. }\end{array}$ & $\begin{array}{l}\mathrm{ETCO}_{2} \\
\mathrm{~mm} \mathrm{Hg}\end{array}$ & $\begin{array}{c}\mathrm{SpO}_{2} \\
(\%)\end{array}$ & $\begin{array}{c}\mathrm{CO}_{2} \\
\mathrm{mmHg}\end{array}$ & $\begin{array}{l}\text { P.A.S. } \\
\mathrm{mmHg}\end{array}$ & $\begin{array}{l}\text { P.A.M } \\
\text { mmHg. }\end{array}$ & $\begin{array}{l}\text { P.A.D. } \\
\mathrm{mmHg}\end{array}$ & $\mathrm{pH}$ & $\begin{array}{c}\mathrm{PaO}_{2} \\
\mathrm{mmHg}\end{array}$ & $\begin{array}{l}\mathrm{PaCO}_{2} \\
\mathrm{mmHg}\end{array}$ & $\begin{array}{l}\text { Temp. } \\
{ }^{\circ} \mathrm{C} \\
\end{array}$ \\
\hline Isoflurano & $\begin{array}{l}117,9^{A} \\
\pm 15,9\end{array}$ & $\begin{array}{l}19,6^{\mathrm{A}} \\
\pm 6,5\end{array}$ & $\begin{array}{l}41,1^{\mathrm{A}} \\
\pm 2,9\end{array}$ & $\begin{array}{l}98,3^{\mathrm{A}} \\
\pm 0,5\end{array}$ & $0 \pm 0$ & $\begin{array}{l}113,9^{A} \\
\pm 15,2\end{array}$ & $\begin{array}{r}72,6^{\mathrm{A}} \\
\pm 13,0\end{array}$ & $\begin{array}{r}54,3^{\mathrm{A}} \\
\pm 11,5\end{array}$ & $\begin{array}{c}7,39^{A} \pm \\
0,007\end{array}$ & $\begin{array}{l}523,2^{A} \\
\pm 45,9\end{array}$ & $\begin{array}{l}41,1^{\mathrm{A}} \\
\pm 3,0\end{array}$ & $\begin{array}{l}37,9^{\mathrm{A}} \\
\pm 0,3\end{array}$ \\
\hline $\begin{array}{c}\text { Isoflurano- } \\
\text { Fentanil }\end{array}$ & $\begin{array}{r}62,2^{\mathrm{B}} \\
\pm 10,2\end{array}$ & $\begin{array}{l}11,7^{\mathrm{B}} \\
\pm 2,5\end{array}$ & $\begin{array}{l}39,9^{\mathrm{B}} \\
\pm 2,4\end{array}$ & $\begin{array}{l}98,2^{A} \\
\pm 0,4\end{array}$ & $0 \pm 0$ & $\begin{array}{r}133,9^{\mathrm{B}} \\
\pm 10,6\end{array}$ & $\begin{array}{r}79,4^{\mathrm{B}} \\
\pm 11,0\end{array}$ & $\begin{array}{r}55,7^{A} \\
\pm 10,8\end{array}$ & $\begin{array}{c}7,38^{\mathrm{B}} \pm \\
0,008\end{array}$ & $\begin{array}{l}516,2^{\mathrm{A}} \\
\pm 46,2\end{array}$ & $\begin{array}{l}40,5^{\mathrm{A}} \\
\pm 2,3\end{array}$ & $\begin{array}{l}38,0^{\mathrm{A}} \\
\pm 0,4\end{array}$ \\
\hline $\begin{array}{c}\text { Isoflurano- } \\
\text { salina }\end{array}$ & $\begin{array}{l}116,8^{\mathrm{A}} \\
\pm 14,9 \\
\end{array}$ & $\begin{array}{r}21,5^{\mathrm{A}} \\
\pm 10,50 \\
\end{array}$ & $\begin{array}{r}41,3 \\
\pm 3,07 \\
\end{array}$ & $\begin{array}{r}98,6^{\mathrm{A}} \\
\pm 0,8 \\
\end{array}$ & $0 \pm 0$ & $\begin{array}{l}117,4^{\mathrm{A}} \\
\pm 12,8 \\
\end{array}$ & $\begin{array}{r}73,6^{\mathrm{A}} \\
\pm 12,6 \\
\end{array}$ & $\begin{array}{l}54,8^{A} \\
\pm 9,4 \\
\end{array}$ & $\begin{array}{c}7,39^{A} \pm \\
0,014\end{array}$ & $\begin{array}{l}531,9^{A} \\
\pm 45,7 \\
\end{array}$ & $\begin{array}{l}39,9^{A} \\
\pm 2,6 \\
\end{array}$ & $\begin{array}{l}37,9^{\mathrm{A}} \\
\pm 0,5 \\
\end{array}$ \\
\hline
\end{tabular}

F.C. = freqüência cardíaca

$F . R .=$ freqüência respiratória

$\mathrm{ETCO}_{2}=$ pressão parcial de $\mathrm{CO}_{2}$ ao final da expiração

$\mathrm{SpO}_{2}=$ saturação de $\mathrm{O}_{2}$ na hemoglobina

$\mathrm{CO}_{2}=\mathrm{CO}_{2}$ mínimo inspirado

P.A.S.= pressão arterial sistólica

P.A.M.= pressão arterial média

P.A.D.= pressão arterial diastólica

$\mathrm{PaO}_{2}=$ pressão parcial de $\mathrm{O}_{2}$ no sangue arterial

$\mathrm{PaCO}_{2}=$ pressão parcial de $\mathrm{CO}_{2}$ no sangue arterial

\section{DISCUSSÃO}

Os fatores que podem alterar a CAM dos anestésicos inalatórios incluem temperatura corporal, hipotensão acentuada, equilíbrio ácido básico, hipoxemia, hipocapnia, hipercapnia, gestação, idade e ritmo circadiano [12,21]. Em nosso estudo, fatores como tem- peratura corporal, hipotensão, $\mathrm{pH}, \mathrm{PaCO}_{2}$ e $\mathrm{PaO}_{2}$ foram levados em consideração e mantidos dentro de uma variação normal que não alteraria a CAM. Como a gestação pode alterar a CAM dos anestésicos inalatórios, os animais utilizados neste estudo foram previamente submetidos a ovariohisterectomia. A faixa etária dos animais utilizados variou entre 2 e 4 anos, evi- 
tando-se com isso, possíveis variações relacionadas com a idade. Com relação às alterações circadianas, que poderiam influenciar na mensuração da CAM, os procedimentos experimentais foram realizados no mesmo horário.

A CAM do isoflurano já foi determinada em cães e os valores médios encontrados foram: 1,28\% [25], 1,31\% [23], 1,30\% [26] e 1,39\% [27]. Em nosso estudo, a CAM do isoflurano observada foi $\bar{X} 1,27 \pm$ 0,02 , semelhante aos valores descritos anteriormente. Esta discreta diferença entre os estudos poderia ser explicada quando a CAM é mensurada em tempos distintos, podendo variar no mesmo animal em torno de $10 \%$. Este fato foi relatado por Eger et al. [2], que ao determinarem a CAM do halotano no mesmo cão durante semanas sucessivas, observaram que a mesma variou $8 \%$ em média e no mesmo animal, no mesmo dia, a variação entre registros sucessivos também foi de $8 \%$. Hellyer et al. [6], determinando a CAM do isoflurano $(1,80 \pm 0,21 \%)$ em 8 cães, onde cada animal foi anestesiado 3 vezes com um intervalo mínimo de 1 semana, também não observaram diferenças significativas nos valores da CAM do isoflurano.

A utilização intra-operatória de opióides previne as respostas autonômicas ao estímulo doloroso resultando em melhor estabilidade hemodinâmica, reduz as exigências de agentes anestésicos intravenosos ou inalatórios como também a dor e o desconforto no pósoperatório [9]. A fentanila foi selecionada neste estudo por ser o opióide mais utilizado como parte de uma técnica anestésica balanceada, com duas finalidades básicas: analgesia e controle de respostas autonômicas ao estímulo doloroso [19]. Além disso, seu perfil farmacocinético [15] e potencial de redução da CAM do isoflurano [18] foram previamente relatados em cães, e seus efeitos farmacológicos correlacionam-se intimamente com as concentrações no plasma [7], permitindo que seja administrada em infusão contínua [16].

O efeito da fentanila sobre a CAM do enflurano $[8,16]$ e do isoflurano [18] foi investigado em cães. Nestes estudos, foi administrada fentanila em bolus (durante 20 minutos) seguido de infusão contínua, sendo a CAM determinada após um período de 60 minutos. Segundo estes autores, este procedimento permite que uma concentração plasmática constante seja atingida. Como em nosso estudo os níveis plasmáticos de fentanil não foram mensurados e com o intuito de obtermos uma concentração plasmática constante, seguimos o preconizado por Murphy \& Hug [16], administrando este fármaco na dose de $30 \mu \mathrm{g} / \mathrm{kg}$ durante 20 minutos seguido por infusão contínua $(0.2 \mu \mathrm{g} / \mathrm{kg} / \mathrm{min})$, sendo a CAM isoflurano-fentanila determinada após um período de 60 minutos.

Segundo Murphy \& Hug [18], a CAM do isoflurano em cães foi reduzida em $63 \pm 2 \%$ após administração de fentanila em bolus $(45 \mu \mathrm{g} / \mathrm{kg}$ durante 20 minutos) e em infusão contínua ( $0.2 \mu \mathrm{g} / \mathrm{kg} / \mathrm{min})$, sendo que uma concentração plasmática maior que $21 \mathrm{ng} / \mathrm{mL}$ não produziu mais que $67 \%$ de redução da CAM do isoflurano. Hellyer et al. [6] observaram uma redução de 52.8\% da CAM do isoflurano após administração de fentanila em bolus ( $10 \mu \mathrm{g} / \mathrm{kg}$ durante 60 segundos) seguido por infusão contínua $(0,3 \mu \mathrm{g} / \mathrm{kg} / \mathrm{min})$, sendo que a concentração plasmática de fentanila aumentou com o tempo e os autores atribuíram, em parte, às altas doses de fentanila utilizadas e ao uso concomitante com isoflurano, contrariando outros trabalhos nos quais a concentração plasmática de fentanila permaneceu relativamente constante durante todo o período de determinação da CAM $[16,18]$. Em nosso estudo, a fentanila diminuiu significativamente $(\mathrm{P}<0,05)$ o valor médio da CAM do isoflurano de $1,27 \pm 0,02 \%$ para $0,73 \pm 0,08 \%$, representando uma redução de $42 \%$, semelhante ao resultado observado por Hellyer et al. [6], quando a CAM do isoflurano foi reduzida de $1,80 \pm 0,21 \%$ para $0,85 \pm$ $0,14 \%$, representando uma redução de $52,8 \%$, apesar de o nosso estudo diferir no bolus, na velocidade de infusão contínua e no período de início da determinação da CAM isofluranoentanila. Não obstante, quando comparamos os resultados do presente trabalho com os de Murphy \& Hug [18], que utilizaram a mesma velocidade de infusão contínua de fentanila, encontramos resultados distintos e de difícil comparação, pois estes autores não citaram os valores da CAM do isoflurano antes e após a administração do fentanila.

Vários estudos indicam que infusão contínua ou bolus múltiplos intermitentes estão associados com o aparecimento de tolerância a fentanila dentro de 180 minutos nos cães e, em humanos, dentro de algumas horas ou dias. Esta tolerância desenvolve-se mais rapidamente quando altas doses são utilizadas ou quando os intervalos entre doses são curtos [1]. Hall et al. [3] observaram o aparecimento de tolerância ao sufentanil administrado em cães através de infusão contínua quando uma redução inicial da CAM de $34 \%$ passou para $18 \%$ após 450 mi- 
nutos a partir do início da infusão. Porém, Murphy \& Hug [16], observaram a mesma redução da CAM do enflurano pela administração de fentanila em infusão contínua durante 520 minutos. Em nosso experimento, o tempo de infusão contínua de fentanila (X 142,71 \pm 9,55 minutos) foi inferior ao tempo de infusão descrito por Askitopoulou et al. [1] e Hall et al. [3], e corrobora o relatado por Murphy \& Hug [16], que não observaram o aparecimento de tolerância à fentanila.

Durante a realização dos experimentos, observou-se, em alguns animais, um acentuado aumento da temperatura corporal e taquipnéia decorrente do mesmo. A utilização de bolsas de gelo sobre o animal fez com que a temperatura corporal retornasse aos valores normais (E. P. Steffey, comunicação pessoal, 1999). Conforme Sackman [22], esta ocorrência devido à administração de fentanila poderia estar relacionada com uma sensibilização auditiva e elevação do centro termorregulador do hipotálamo ocasionando taquipnéia.

Apesar de os objetivos não incluírem os efeitos de uma velocidade de infusão contínua de fentanila em cães anestesiados com isoflurano, é válido mencionar que todos os parâmetros hemodinâmicos e hemogasométricos permaneceram dentro dos limites de normalidade, estando de acordo com os resultados obtidos por Ilkiw et al. [8], que observaram os efeitos ácido-básico e cardiovasculares durante infusão contínua de fentanila $(1,065 \mu \mathrm{g} / \mathrm{kg} / \mathrm{min})$ em cães anestesiados com enflurano.

\section{CONCLUSÃO}

A administração de fentanila em infusão contínua $(0,2 \mu \mathrm{g} / \mathrm{kg} / \mathrm{min})$ nos cães deste estudo reduziu a CAM do isoflurano em $42 \%$, permitindo uma redução na necessidade do agente anestésico durante o período transanestésico, além de demonstrar que a utiliza- ção deste fármaco produziu uma adequada estabilidade cardiovascular.

\section{NOTAS INFORMATIVAS}

${ }^{1}$ Isoflurano - Isoflurane (Abbott Laboratórios do Brasil Ltda., São Paulo, SP, Brasil)

${ }^{2}$ Máscara facial (Calgimed Equipamentos para Eletromedicina e Engenharia Ltda., São Paulo, SP, Brasil)

${ }^{3}$ Circuito valvular com absorção de $\mathrm{CO}_{2}$ ( $\mathrm{HB}$ - Hospitalar Indústria e Comércio Ltda., Vila Friburgo, SP, Brasil)

${ }^{4}$ Vaporizador calibrado para isoflurano (HB 4.4 Abbott Laboratórios do Brasil Ltda., São Paulo, SP, Brasil)

${ }^{5}$ Monitor e Transdutor Biomedical Systems Inc. 404 Patient Monitor (Hewlett Packard, EUA)

${ }^{6}$ Analisador de gases sanguíneos (Mod AGS 21 Drake, São Paulo, Brasil)

${ }^{7}$ Colchão térmico (Vector Thermal Barrier - Model $n^{\circ}$ 20s/n 5020, Colorado, EUA)

${ }^{8}$ Termômetro eletrônico digital (Digicare TTE II Biomedical Technology INC, Palm Beach, Florida, EUA).

${ }^{9}$ Eletrocardioscópio (Drake, Mod MDK 2007, São Paulo, Brasil)

${ }^{10}$ Pulso oxímetro (Ohmeda Mod - 3700, Louisville, EUA)

${ }^{11}$ Analisador de gases exalados (Multigas Monitor 9100 - BCI International, Waukesha, Wis, EUA)

${ }^{12}$ Citrato de fentanil - Fentanil (Abbott Laboratórios do Brasil Ltda., São Paulo, SP, Brasil)

${ }^{13}$ Bomba de infusão contínua - Digipump SR 2000 (Bomba de infusão de seringa - Digicare Tecnologia Biomédica Ltda, Rio de Janeiro, RJ, Brasil).

${ }^{14}$ Estimulador elétrico - MGF II - Medeir (Medical Cirúrgica Ltda., São Paulo, SP, Brasil). E-mail: epsteffey@ucdavis.edu

\section{REFERÊNCIAS}

1 Askitopoulou H., Whitwam J.G., Al-Khudhairi D., Chakrabarti M., Bower S. \& Hull C.J. 1985. Acute Tolerance to Fentanyl During Anesthesia in Dogs. Anesthesiology. 63: 255-261.

2 Eger E. I., Saidman L. \& Brandstater B. 1965. Minimum Alveolar Concentration: A Standard of Anesthetic Potency. Anesthesiology. 26: 756-763.

3 Hall R. I., Murphy M. R. \& Hug C. C. 1987. The Enflurane Sparing Effect of Sufentanil in Dogs. Anesthesiology. 67:518-525.

4 Hall R. I., Szlam F. \& Hug C. C. 1987. The Enflurane Sparing Effect of Alfentanil in Dogs. Anesthesia and Analgesia. 66: 1287-1291.

5 Hellyer P. W. 1997. Management of Acute and Surgical Pain. Seminars in Veterinary Medicine and Surgery (Small Animal). 12: 106-114.

6 Hellyer P. W., Mama K. R., Shafford H. L., Wagner A. E. \& Kollias-Baker C. 2001. Effects of Diazepam and Flumazenil on Minimum Alveolar Concentrations for Dogs Anesthetized With Isoflurane or a Combination of Isoflurane and Fentanil. American Journal of Veterinary Research. 62: 555-560. 
7 Hug C. C. \& Murphy M. R. 1979. Fentanyl Disposition in Cerebrospinal Fluid and Plasma ans Its Relationship to Ventilatory Depression in the Dog. Anesthesiology. 50: 342-349.

8 Ilkiw J. E., Pascoe P. J., Haskins S. C., Patz J. D. \& Jaffe R. 1993. The Cardiovascular Sparing Effect of fentanyl and Atropine, Administered to Enflurane Anesthetized Dogs. Canadian Journal of Veterinary Research. 58: 248-253.

9 Ilkiw J. E. 1999. Balanced Anesthetic Techniques. Clinical Techniques in Small Animal Practice. 14: 27-37.

10 Lake C. L., Difazio C. A., Mosciki J. C. \& Engle J. S. 1985. Reduction in Halotane MAC: Comparison of Morphine and Alfentanil. Anesthesia and Analgesia. 64: 807 - 810.

11 Lumb W. V. \& Jones E. W. 1984. Preanesthetic Agents. In: Veterinary Anesthesia. 2nd edn. Philadelphia: Lea \& Febiger, pp.65- 197.

12 Lumb W. V. \& Jones E. W. 1996. Considerations for General Anesthesia. In: Veterinary Anesthesia. 3rd edn. Philadelphia: Lea \& Febiger, pp.5-34.

13 Marshall B. E. \& Longnecker D. E. 1994. Anestésicos Gerais. In: Gilman, A. G. (Ed). Goodman \& Gilman: As Bases Farmacológicas da Terapêutica. 8.ed. Rio de Janeiro: Editora Guanabara Koogan, pp.189-204.

14 McEwan A. I., Smith C., Dyar O., Goodman D., Smith L. R. \& Glasas P. S. A. 1993. Isoflurane Minimum Alveolar Concentration Reduction by Fentanyl. Anesthesiology. 78: 864-869.

15 Murphy M. R., Olson W. A. \& Hug C. C. 1979. Pharmacokinetics of H-Fentanyl in the Dog Anesthetized with Enflurane. Anesthesiology. 50: 13-19.

16 Murphy M. R. \& Hug C. C. 1982. The Anesthetic Potency of Fentanil in Therms of its Redution of Enflurane MAC. Anesthesiology. 57: 485-488.

17 Murphy M. R. \& Hug C. C. 1982. The Enflurane Sparing Effect of Morphine, Butorphanol and Nalbuphine. Anesthesiology. 57: 489-492.

18 Murphy M. R. \& Hug C. C. 1983. Efficacy of Fentanyl in Reducing Isoflurane MAC; Antagonism by Naloxone and Nalbuphine. Anesthesiology. 59: A338.

19 Nocite J. R. 1993. Opiácios: Benefícios e Riscos na Anestesia, na Dor e nos Cuidados Intensivos. Revista Brasileira de Anestesiologia. 43: 277-282.

20 Poklis A. 1995. Fentanyl: A Review for Clinical and Analytical Toxicologists. Clinical Toxicology. 33: 439-447.

21 Quasha A. I., Egger E. I. \& Tinker J. H. 1980. Determination and Applications of MAC. Anesthesiology. $53: 315$ - 334.

22 Sackman J. E. 1991. Control Pain in Animals. Continuing Education Article. 13: 181-189.

23 Schwieger I. M., Szlam F. \& Hug C. C. 1989. Absence of Agonistic or Antagonistic Effect of Flumazenil in Dogs Anesthetized with Enflurane, Isoflurane or Fentanyl-Enflurane. Anesthesiology. 70: 477-480.

24 Stanley T. H. 1992. The History and Development of the Fentanyl Series. Journal of Pain Symptom Management.7: 3-7.

25 Steffey E. P. \& Howland Jr. D. 1977. Isoflurane Potency in the Dog and Cat. American Journal of Veterinary Research. 38: 1833-1836.

26 Steffey E. P., Baggot J. D., Eisele J. H., Willitss N., Wouliner M. J., Jarvis K. A., Elliot A. R. \& Tagawa M. 1994. Morphine-Isoflurane Interaction in Dogs, Swine and Rhesus Monkeys. Journal of Veterinary Pharmacology and Therapeutics. 17: 202-210.

27 Steffey E. P., Eisele J. H., Baggot J. D., Wouliner M. J., Jarvis K. A. \& Elliot A. R. 1993. Influence of Inhaled Anesthetics on the Pharmacokinetics and Pharmacodynamics of Morphine. Anesthesia and Analgesia. 77: $346-351$.

28 Vianna P. T. G., Lapa J. J. B., Ganem E. M. \& Castiglia Y. M. M. 1998. Redução da Concentração Alveolar Mínima do Isoflurano e do Sevoflurano pelo Fentanil. Revista Brasileira de Anestesiologia. 48: 161-168. 